\title{
Influence of exhaled breath condensates from children with asthma on endothelial cells cultured in vitro. Do we really know everything about our breath condensate?
}

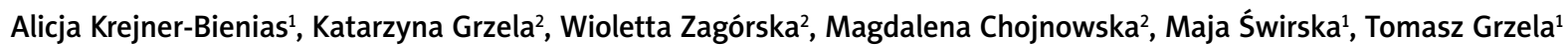 \\ ${ }^{1}$ Department of Histology and Embryology, Medical University of Warsaw, Warsaw, Poland \\ 2Department of Paediatrics, Pneumonology and Allergology, Medical University of Warsaw, Warsaw, Poland \\ Adv Dermatol Allergol 2020; XXXVII (6): 968-974 \\ DOI: https://doi.org/10.5114/ada.2019.86181
}

\begin{abstract}
Introduction: Asthma-associated remodelling involves subepithelial fibrosis and increased vascularization of the bronchial wall. The latter may be associated with excessive production of several angiogenesis regulators which may be found in exhaled breath condensates (EBCs) collected from children with asthma.

Aim: To assess the influence of EBC samples of asthmatic children and healthy controls on in vitro cultures of normal human lung microvascular endothelial cells (HLMVEC) and murine endothelial cell line (C-166). Moreover, the proteomic profile of cytokines in EBC samples was analysed.

Material and methods: Breath condensates collected from children with mild asthma $(n=10)$ and from healthy controls $(n=10)$ were used for experiments. Colorimetric tetrazolium salt reduction assay was used to evaluate the effect of EBCs on HLMVEC and C-166 cell lines. Furthermore, influence of EBCs on C-166 cell line was assessed using Annexin V-binding assay. The cytokine screening of EBC samples was performed using a proteome microarray system. Results: The EBCs from patients with asthma revealed a weak inhibitory influence on human and murine endothelial cells. Surprisingly, EBCs from healthy children led to cell death, mainly by the induction of apoptosis. There were no statistically significant differences in the cytokine profile between EBC samples from children with asthma and healthy controls.

Conclusions: Our preliminary report shows for the first time that the incubation of EBCs from healthy controls induced apoptosis in endothelial cells. The detailed mechanism responsible for this action remains unknown and requires further research.
\end{abstract}

Key words: angiogenesis, asthma, endothelial cells, breath condensate, MTT.

\section{Introduction}

The respiratory tract in asthma undergoes some structural changes, which are better known as remodelling. Airway wall remodelling includes subepithelial fibrosis, hyperplasia and hypertrophy of airway smooth muscle cells, and also an increased vascularization and angiogenesis in submucosa [1]. The latter two could be related to an imbalance between some angiogenesis regulators, mainly vascular endothelial growth factor (VEGF) and angiopoietin-1 [2]. Elevated levels of angiogenic factors in the bronchial wall of asthmatic patients have been shown in several studies [1, 3]. The presence of the angiogenesis-related factors was investigated also in exhaled breath condensates (EBCS) of children with asthma [4].
The collection of exhaled breath condensate is a novel, non-invasive research and diagnostic tool, which requires minimal patient cooperation $[5,6]$. The biochemical composition of condensate samples reflects, at least to some extent, the current pathophysiological status of respiratory tract [7]. Therefore, EBC analysis may be useful in diagnosing of various pathologies, including asthma, especially in younger children and patients with exacerbation of the disease, who are unable to perform other diagnostic tests [6]. The assessment of the presence and concentration of various biomarkers of oxidative stress and inflammation, including eicosanoids, cytokines, chemokines and growth factors, in EBC samples [8] seems to be a promising way for both understanding of

\footnotetext{
Address for correspondence: Tomasz Grzela MD, PhD, Department of Histology and Embryology, Medical University of Warsaw, 5 Chalubinskiego St, 02-004 Warsaw, Poland, e-mail: tomekgrzela@gmail.com Received: 7.05.2019, accepted: 27.05.2019. 
asthma pathophysiology and easy, non-invasive monitoring of the disease course.

In a recent study we have demonstrated that EBC samples contained significant quantities of several angiogenesis regulators [4]. Moreover, it was suggested that the amount of angiogenesis-related factors in EBC as well as vascular density of the airway wall may correlate with asthma severity $[1,4]$. On the other hand, as mentioned previously, EBCs from asthma patients may contain a mixture of pro-inflammatory and immunoregulatory cytokines, which can modify the influence of angiogenesis regulators. However, most of studies focused on assessment of only a few selected cytokines, mainly due to the fact that broad cytokine screening with most commonly used enzyme-linked immunosorbent assays (ELISA) was limited by large quantities of material necessary for each cytokine. Hence, it would be beneficial to use a method which enables parallel detection of multiple proteins in small amount of EBC in a single experiment. It has been proven that aforementioned requirements are fulfilled by the proteome microarray, which may be used for fast and broad semi-quantitative screening of multiple proteins, e.g. immunoregulatory cytokines, in EBC samples.

\section{Aim}

The aim of our proof-of-concept study was to verify whether previously mentioned regulators of angiogenesis in EBC samples, collected from children with asthma or healthy controls, may influence in vitro cultures of endothelial cells. Moreover, we assessed the profile of proinflammatory and immunoregulatory cytokines in breath condensates in order to compare expected differences between samples from asthmatic and healthy children.

\section{Material and methods}

\section{EBC samples collection and preparation}

The already deposited exhaled breath condensate samples, excessively collected for our recent projects [4, $9,10]$, according to approval (KB/246/2012) of the Bioethics Committee at the Medical University of Warsaw, were used for in vitro experiments. Ten samples originated from children with well-controlled chronic allergic asthma. The control group comprised 10 samples of healthy individuals. The characteristics of patients and control individuals were already described in previous publications [4]. The EBC samples were aliquoted and stored at $-70^{\circ} \mathrm{C}$, to avoid repeated freezing-thawing cycles.

\section{Cell cultures}

The Human Lung Microvascular Endothelial Cells, passages $6^{\text {th }}$ to $10^{\text {th }}$ (HLMVEC, Cell Applications, Inc., San Diego, CA) and murine endothelial cell line (C-166) were used for in vitro studies. The cells were maintained in
Endothelial Cell Growth Medium (ECGM, Cell Applications, Inc., San Diego, CA), in $25 \mathrm{~cm}^{2}$ culture flasks under standard conditions of $5 \% \mathrm{CO}_{2}$ in humidified air, at $37^{\circ} \mathrm{C}$.

\section{MTT reduction assay}

Cells were trypsinized from subconfluent (approximately $80 \%$ ) cultures, resuspended in fresh culture medium and seeded in triplicates onto 96-well flat bottom culture plates, at a density of 10,000 cells/well, in $100 \mu \mathrm{l}$. After $4 \mathrm{~h}$ to allow a cell attachment to the plastic, $25 \mu \mathrm{l}$ of EBC samples or test controls were added to respective wells. Test controls included $25 \mu$ of ECGM culture medium (baseline control), 0.1\% Triton X-100 (negative control) and sterile double distilled water (solvent control). Then, the plates were incubated for next $20 \mathrm{~h}$ at $37^{\circ} \mathrm{C}$. Afterwards, $20 \mu \mathrm{l}$ of 3-(4,5-dimethylthiazol-2-yl)-2,5diphenyltetrazolium bromide (MTT, from SIGMA-Aldrich, St. Louis, MO) in PBS were added to each well, to the final concentration of $0.3 \mathrm{mg} / \mathrm{ml}$, followed by $4 \mathrm{~h}$ of incubation. At the end of incubation the cultures were inspected using the phase contrast microscope (Zeiss Primovert, from Carl Zeiss Microscopy GmbH, Gottingen, Germany) and their pictures were taken at 400× magnification.

Finally, after short centrifugation, supernatants were carefully removed and cells were lysed in $200 \mu \mathrm{l}$ of dimethyl sulfoxide (DMSO, SIGMA-Aldrich Chemie GmbH, Steinheim, Germany) with $\mathrm{HCl}$. Absorbance of cell lysates was measured at $\lambda=540 \mathrm{~nm}$, using Microplate Reader 550 (BIO-RAD, Hercules, CA). MTT reduction by target cells in a presence of tested EBC samples was expressed as the percent of counts from baseline control (cells with medium alone), which was considered as 100\%. Counts from negative control (cells treated with Triton X-100) were considered as $0 \%$.

All tests were repeated three times, and their results were calculated and compared using Mann-Whitney $U$ test. The differences between groups were considered statistically significant at $p<0.05$.

\section{Annexin V-binding assay}

The influence of EBC samples on murine endothelial C-166 cell line was also assessed using FITC (fluorescein isothiocyanate)-labelled Annexin V/propidium iodine (PI) assay. The test was performed according to a modified manufacturer's protocol (FITC Annexin V Apoptosis Detection Kit, BD Pharmingen, Franklin Lakes, NJ) [11]. The cells were incubated with medium alone (baseline control), EBC samples or $0.02 \%$ Triton X-100 (negative control) for 1 or $4 \mathrm{~h}$. Then, they were subsequently washed twice with cold PBS and incubated with FITC-labelled Annexin $\mathrm{V}$ and $\mathrm{PI}$ for $15 \mathrm{~min}$. The test was repeated three times. The presence of Annexin V-and/or PI-positive cells was assessed using Nikon Eclipse E800 fluorescent microscope with Nikon Coolpix 995 digital camera (Nikon, Japan). 


\section{Cytokine microarray}

The assay was performed using Proteome Profiler TM Human XL Cytokine Array Kit (R\&D Systems Inc., Minneapolis, MN) according to a detailed protocol provided by the manufacturer. In brief, $400 \mu$ l of randomly selected breath condensates from children with asthma and healthy controls (2 samples from each group) were applied onto nitrocellulose membranes spotted with respective capture antibodies. After overnight incubation at $4^{\circ} \mathrm{C}$, the membranes were washed and incubated with streptavidin-horseradish peroxidase conjugate. Then, stabilized luminol was added on membranes, followed by their 10-15 min exposure in the chemiluminescence detection scanner (FluorChem E system, ProteinSimple, San Jose, CA). The membrane scans were subjected to further densitometric assessment using ImageJ software 1.51p (National Institutes of Health scientific image-analysis program), as previously described $[12,13]$. The mean count of each analyte was compared to mean intensity of positive control/reference spots and expressed as a sample-to-reference control ratio $(x / R)$. Statistical analysis was performed using Student $t$ test and analysis of variance (ANOVA). Values of $p<0.05$ were considered statistically significant.

\section{Results}

\section{MTT reduction assay}

Both human and murine endothelial cells, when incubated with EBC samples from children with asthma, revealed a significantly lower level of formazan (insoluble product of MTT reduction), as compared to respective cultures in medium alone (Figure $1 \mathrm{~A}$ ). As observed in the phase contrast microscope, the number of cells incubated with asthmatics' condensates was markedly lower than that of respective cells cultured in medium alone. Interestingly, specific MTT-reducing activity of individual cells, cultured in medium with or without asthmatics' $\mathrm{EBC}$ was very similar (Figures $1 \mathrm{~B} a-c, \mathrm{C} a-c$ ).

Unexpectedly, both HLMVEC and C-166 endothelial cells, when incubated with EBC samples from healthy individuals, displayed a very low level of insoluble formazan (Figure $1 \mathrm{~A}$ ). When inspected in the phase contrast microscope, all cells in these cultures were damaged, with intense debris formation. Surprisingly, this effect was similar to that observed in cells incubated with Triton X-100 (negative control). There was not observed any specific, MTT-reducing activity in both tested endothelial cell cultures, after incubation with 8 of 10 EBC samples from healthy individuals (Figures $1 B d-f, C d-f$ ). The remaining two $E B C$ samples from the control subjects group revealed a less pronounced effect on HLMVEC cells, but, in case of C-166 cell line, that effect was similar to other condensates from that group (Figure $1 \mathrm{~A}$ ). Furthermore, the observed detrimental effect of conden- sates from healthy subjects on in vitro cultured human and murine endothelial cells was dose dependent (data not shown). Noteworthy, cells incubated with addition of distilled water (solvent control) revealed the formazan level similar to that of cells with culture medium (data not shown).

\section{Annexin V-binding assay}

The incubation of C-166 cells with exhaled breath condensates of children with asthma did not reveal any noticeable change in baseline fluorescence, which would be observed with FITC-, or PI-specific filters until the end of the experiment (data not shown). In contrast, 1-hour incubation of C-166 endothelial cells with EBC samples from healthy individuals induced a mild increase in $\mathrm{PI}$ mediated red fluorescence of all cells, but also a visible increase in FITC-mediated green fluorescence observed in approx. $22 \%$ of cells. After 4 -hour incubation the further enhancement of green fluorescence was observed in all cells. All cells incubated with Triton X-100 (negative control) revealed strong $\mathrm{Pl}$-mediated red fluorescence, but they were all FITC-negative (Figure 2).

\section{Cytokine microarray}

The densitometric analysis of the cytokine profile of all assessed breath condensates did not show any significant difference between both groups. In particular, there was no difference in clusters of pro-inflammatory or immunoregulatory cytokines (Figure 3).

\section{Discussion}

Asthma-associated remodelling is a long-lasting process of unclear origin, which leads to irreversible airway narrowing. It is characterised by the activation and proliferation of smooth muscle cells and myofibroblast, deposition of extracellular matrix components and thickening of the reticular basement membrane [14]. In addition, increased vasculature with angiogenic sprouting is often observed in the asthmatic airway wall [15]. However, the detailed role of angiogenesis in asthma-associated remodelling remains unclear.

Therefore, there is a need for better understanding of this process, which would enable its better control and development of new therapeutic strategies [16]. This issue may be especially important, since anti-inflammatory treatment with inhaled corticosteroids, although effective in modulation of a local inflammatory response, in some cases does not prevent asthma-associated remodelling [17]. Thus, the postulated role of chronic inflammation in this phenomenon seems to be at least controversial. Our previous study has shown that breath condensates from asthmatic children contained a broad spectrum of angiogenesis-related factors. Some of them may also be involved in regulation of airway remodelling 


\section{A}

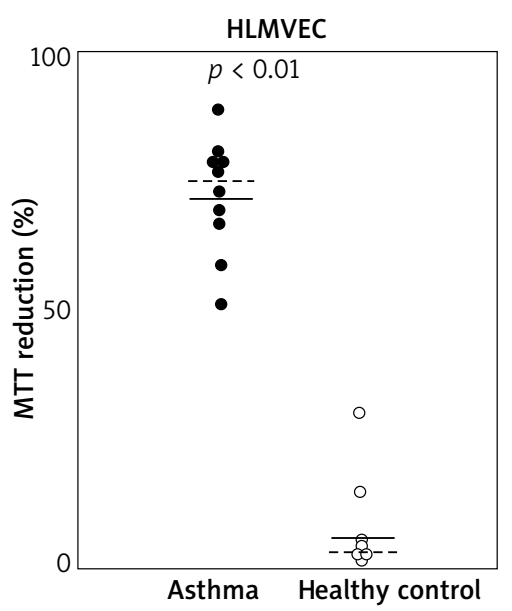

B

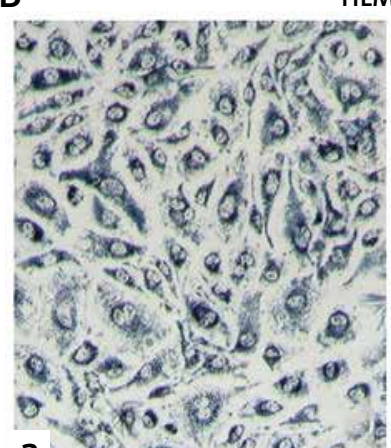

HLMVEC

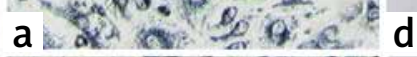
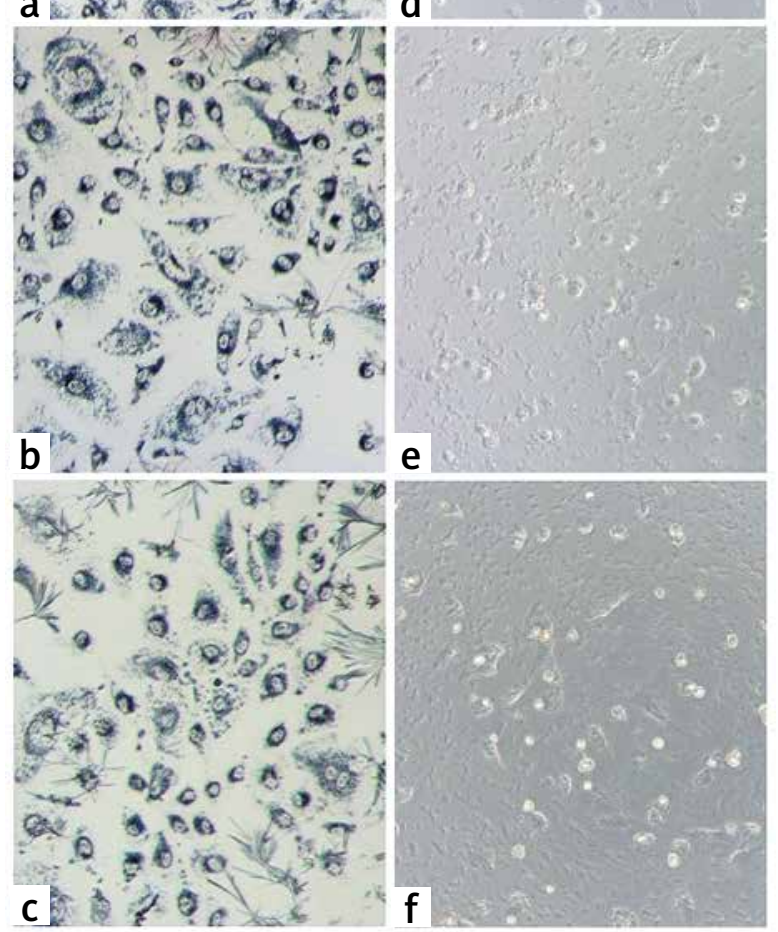

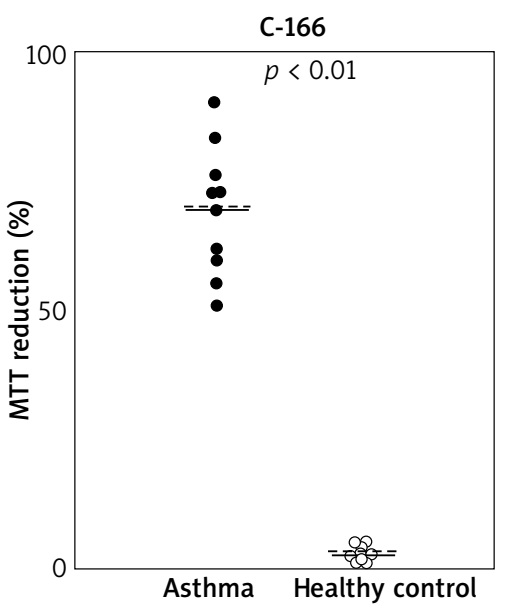

C
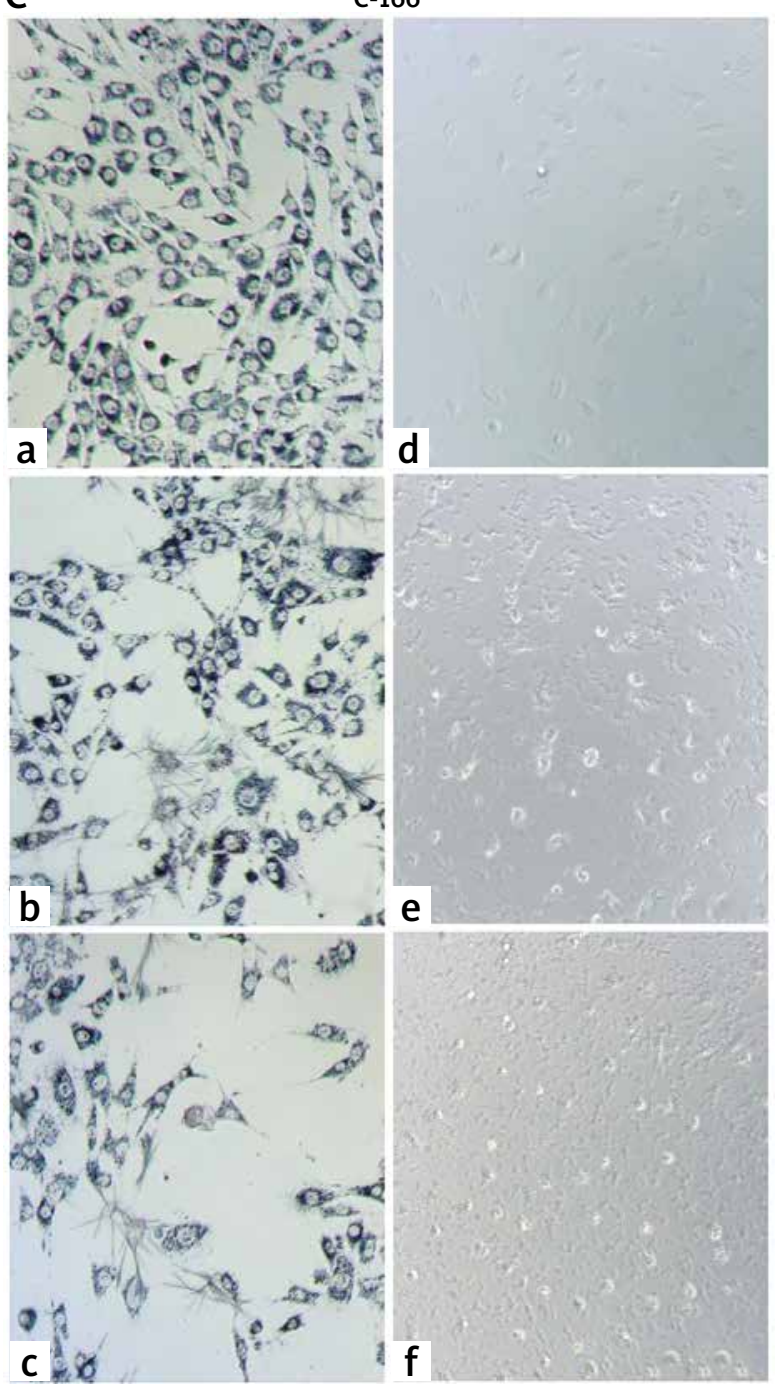

Figure 1. A - MTT reduction by target cells (HLMVEC - left graph, C-166 - right graph) in a presence of EBC samples was expressed as a percentage of counts from baseline control (considered as 100\%). Each dot represents the result of an individual from the asthma or healthy control group. Mean values of the percentage of MTT reduction in each group were indicated as solid lines, the median values were shown as dashed lines. The $p$-values below 0.05 were considered as statistically significant (by Mann-Whitney $U$ test). B - Human lung endothelial cells (HLMVEC), and C - murine endothelial cell line (C-166) after $24 \mathrm{~h}$ (including $4 \mathrm{~h}$ with MTT) incubation with representative EBC samples: a - cells in culture medium (baseline control); $\mathbf{b}$ - cells with EBC from asthma patient \#A1; $\mathbf{c}$ - cells with EBC from asthma patient \#A4; $\mathbf{d}$ - cells with Triton X-100 (negative control); e - cells with EBC from healthy control \#K2; $\mathbf{f}$ - cells with EBC from healthy control \#K3 

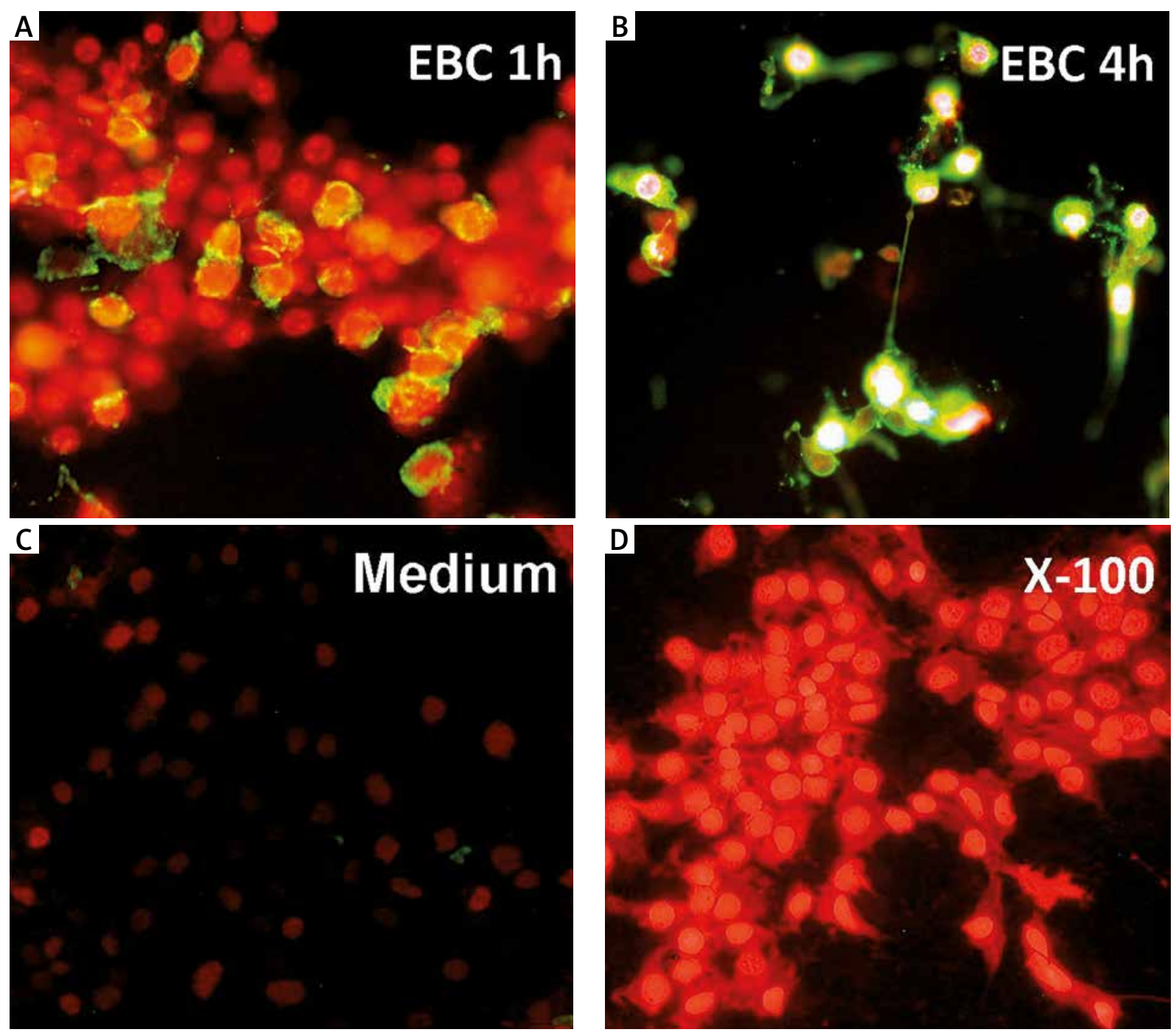

Figure 2. Annexin V-binding by C-166 murine endothelial cell line. The effect of EBC from healthy children on cultured C-166 cells after 1 -hour incubation (EBC $1 \mathrm{~h}, \mathrm{~A}$ ) or after 4-hour exposure (EBC $4 \mathrm{~h}, \mathrm{~B})$. Cells incubated in medium alone (Medium, C) or with $0.02 \%$ Triton (X-100, D) served as controls. Representative areas were photographed using Nikon Eclipse E800 fluorescent microscope equipped with Nikon Coolpix 995 digital camera under 500× magnification

[4]. Hence, the current proof-of-concept study aimed to verify whether $E B C$ samples from children with asthma will stimulate in vitro proliferation of normal human lung microvascular endothelial cells as well as murine endothelial cell line - C-166. For that purpose we have used the colorimetric MTT reduction assay, which enables the assessment of cell metabolic activity and, indirectly, cell proliferation and cytotoxicity [18]. Surprisingly, we have found that the amount of formazan, the insoluble product of MTT reduction, was lower in cultures of endothelial cells with asthmatic EBC, than in cells with medium alone. It reflected a lower number of cells in culture with EBC, presumably due to inhibition of their proliferation, since individual metabolic activity of those cells was very similar to that of endothelial cells with medium alone. This observation was in contrary to our expectations, especially due to increased levels of angiogenin and VEGF, two potent inducers of endothelial cells proliferation, which were found in tested EBC samples [4]. Nevertheless, the most astonishing was the result of incubation of both human and murine endothelial cells, with EBC samples from healthy individuals, which led to endothelial cell death. It was due to induction of apoptosis, as found in Annexin V-binding assay. The EBC samples from healthy children induced apoptosis in C-166 endothelial cells within short, 1-hour incubation. Interestingly, no such influence was observed in EBCs from patients with asthma. 
A

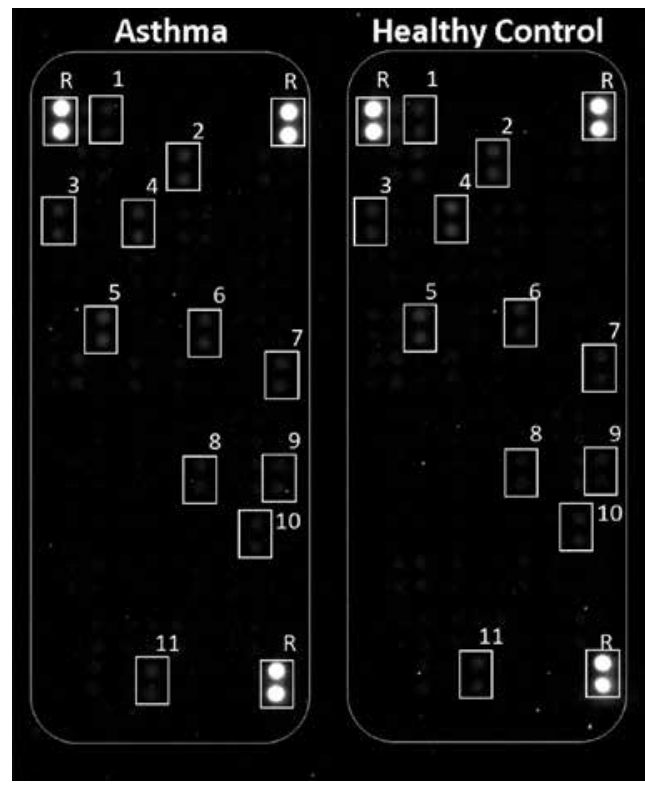

B

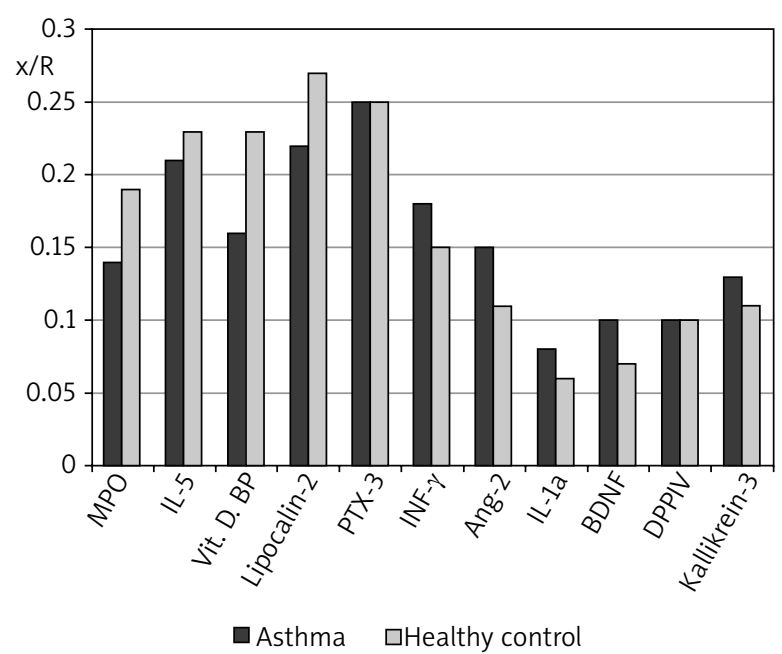

MPO - myeloperoxidase, IL-5 - interleukin-5, Vit. D. BP - vitamin D binding protein, PTX-3 - pentraxin-3, INF- $\gamma$ - interferon $\gamma$, Ang-2 - angiopoietin-2, BDNF 7 - brain-derived neurotrophic factor, DPPIV - dipeptidyl-peptidase IV.

Figure 3. Representative results of cytokine profile assessment. A - The membranes of Human Cytokine Array XL exposed to EBC samples from patients with asthma or healthy controls. Each pair of spots in selected boxes represents a respective target molecule - the numbers in the boxes correspond to respective numbers on graph $\mathrm{B}$. The brightest spots correspond to reference markers (R). B - The graph represents selected cytokines/factors with the highest values of sample-to-reference control ratio $(x / R)$

We speculate that these peculiarities may be explained based on our previous data, regarding the presence of angiogenesis regulators in exhaled breath condensates. It is plausible that the observed effect of EBC may be due to significant amounts of thrombospondin (TSP)-1 in these samples. TSP-1 is a well-known inhibitor of angiogenesis. If working alone, it may be responsible for strong cytotoxic action of EBCs from healthy subjects against endothelia [19]. However, in case of condensates from patients with asthma, their action seemed to be derivative of both, pro- (i.e. ANG, VEGF, etc.) and anti-angiogenic (e.g. TSP-1) factors. Thus, the outcome would depend on predominance of one of aforementioned groups of proteins.

To assess the possible role of such modulators we analysed the profile of 105 immunoregulatory/pro-inflammatory cytokines in EBC samples from children with asthma and from healthy controls. Surprisingly, we did not find any statistically significant differences in cytokine profiles between both groups. One can speculate that at least the concentrations of some pro-inflammatory cytokines (e.g. IL-6, IL-8, TNF) should be elevated in breath condensates from patients with asthma. However, the study group comprised children with mild, well-controlled asthma, and thus, the observed lack of increased aforementioned cytokines may be explained by effective anti-inflammatory treatment with inhaled corticosteroids [20]. Therefore, we can assume that the composition of pro-inflammatory and immunoregulatory cytokines in breath condensates does not play a significant role in the observed pro-apoptotic effect of EBCs from healthy individuals.

On the other hand, possibly, breath condensates from healthy individuals may contain some other, so far unidentified factor/factors, which could play a key role in control of cell proliferation and respiratory tract remodelling. Hence, since identification of such factor would be useful for development of a more effective therapy of asthma, it requires further extensive studies.

\section{Conclusions}

Our report has shown for the first time that exhaled breath condensates from patients with asthma had a weak influence on human and murine endothelial cells, whereas EBCs from healthy children revealed strong pro-apoptotic activity against these cells. However, the detailed mechanism responsible for the effect observed remains to be clarified.

\section{Acknowledgments}

The study was supported by an individual research grant of the Medical University of Warsaw (1M15/ NM6/2018). 


\section{Conflict of interest}

The authors declare no conflict of interest.

\section{References}

1. Keglowich LF, Borger P. The three A's in asthma - Airway smooth muscle, Airway remodeling \& Angiogenesis. Open Respir Med J 2015; 9: 70-80.

2. Makinde T, Murphy RF, Agrawal DK. Immunomodulatory role of vascular endothelial growth factor and angiopoietin-1 in airway remodeling. Curr Mol Med 2006; 6: 831-41.

3. Hoshino M, Takahashi M, Aoike N. Expression of vascular endothelial growth factor, basic fibroblast growth factor, and angiogenin immunoreactivity in asthmatic airways and its relationship to angiogenesis. J Allergy Clin Immunol 2001; 107: 295-301.

4. Grzela K, Litwiniuk M, Krejner A, et al. Increased angiogenic factors in exhaled breath condensate of children with severe asthma - new markers of disease progression? Respir Med 2016; 118: 119-21.

5. Horváth I, Hunt J, Barnes PJ, et al. Exhaled breath condensate: methodological recommendations and unresolved questions. Eur Respir J 2005; 26: 523-48.

6. Szefler SJ, Chmiel JF, Fitzpatrick AM, et al. Asthma across the ages: knowledge gaps in childhood asthma. J Allergy Clin Immunol 2014; 133: 3-13.

7. Aldakheel FM, Thomas PS, Bourke JE, et al. Relationships between adult asthma and oxidative stress markers and $\mathrm{pH}$ in exhaled breath condensate: a systematic review. Allergy 2016; 71: 741-57.

8. Thomas PS, Lowe AJ, Samarasinghe P, et al. Exhaled breath condensate in pediatric asthma: promising new advance or pouring cold water on a lot of hot air? A systematic review. Pediatr Pulmonol 2013; 48: 419-42.

9. Grzela K, Zagórska W, Krejner A, et al. Inhaled corticosteroids do not reduce initial high activity of matrix metalloproteinase (MMP)-9 in exhaled breath condensates of children with asthma exacerbation: a proof of concept study. Cent Eur J Immunol 2016; 41: 221-7.

10. Grzela K, Zagórska W, Krejner A, et al. Polymorphic variants $279 R$ and $668 \mathrm{Q}$ augment activity of matrix metalloproteinase-9 in breath condensates of children with asthma. Arch Immunol Ther Exp 2017; 65: 183-7.

11. Rahden-Staroń I, Grosicka-Maciąg E, Kurpios-Piec D, et al. The effects of sodium diethyldithiocarbamate in fibroblasts V79 cells in relation to cytotoxicity, antioxidative enzymes, glutathione, and apoptosis. Arch Toxicol 2012; 86: 1841-50.

12. Girish V, Vijayalakshmi A. Affordable image analysis using NIH Image/ImageJ. Indian J Cancer 2004; 41: 47.

13. Schneider CA, Rasband WS, Eliceiri KW. NIH Image to ImageJ: 25 years of image analysis. Nat Methods 2012; 9: 671-5.

14. Westergren-Thorsson G, Larsen K, Nihlberg K, et al. Pathological airway remodeling in inflammation. Clin Respir J 2010; 4 Suppl 1: 1-8.

15. Hashimoto M, Tanaka H, Abe S. Quantitative analysis of bronchial wall vascularity in the medium and small airways of patients with asthma and COPD. Chest 2005; 127: 965-72.

16. Saglani S, Lloyd CM. Novel concepts in airway inflammation and remodelling in asthma. Eur Respir J 2015; 46: 1796-804.

17. Bisgaard H, Hermansen MN, Loland L, et al. Intermittent inhaled corticosteroids in infants with episodic wheezing. N Engl J Med 2006; 354: 1998-2005.

18. Morgan DM. Tetrazolium (MTT) assay for cellular viability and activity. Methods Mol Biol 1998; 79: 179-83.
19. Zhao C, Isenberg JS, Popel AS. Human expression patterns: qualitative and quantitative analysis of thrombospondin-1 under physiological and pathological conditions. J Cell Mol Med 2018; 22: 2086-97.

20. Grzela K, Zagorska W, Krejner A, et al. Prolonged treatment with inhaled corticosteroids does not normalize high activity of matrix metalloproteinase-9 in exhaled breath condensates of children with asthma. Arch Immunol Ther Exp 2015; 63: 231-7. 